\title{
EXPERT REVIEWS \\ European Vaccine Initiative: lessons from developing malaria vaccines
}

Expert Rev. Vaccines 10(12), 1697-1708 (2011)

\section{Mark J Geels', Egeruan B Imoukhuede', Nathalie Imbault ${ }^{1}$, Harry van Schooten', Terry McWade², Marita Troye- Blomberg ${ }^{3}$, Roland Dobbelaer ${ }^{4}$, Alister G Craig $^{5}$ and Odile Leroy*1}

'European Vaccine Initiative, UniversitätsKlinikum Heidelberg, Im Neuenheimer Feld 326-3. OG, 69120 Heidelberg, Germany ${ }^{2}$ Royal College of Surgeons in Ireland, 123 St Stephen's Green, Dublin, Ireland ${ }^{3}$ Department of Immunology, Wenner Gren Institute, Stockholm University, SE-10691, Stockholm, Sweden

${ }^{4}$ Waasmunsterbaan 26, B-9160, Lokeren, Belgium

5Liverpool School of Tropical Medicine, Pembroke Place, Liverpool, L3 5QA, UK *Author for correspondence:

Tel.: + 496221565974

Fax: +496221565727 odile.leroy@euvaccine.eu

For over 10 years, the European Vaccine Initiative (EVl; European Malaria Vaccine Initiative until 2009) has contributed to the development of 24 malaria candidate vaccine antigens with 13 vaccine candidates being advanced into Phase I clinical trials, two of which have been transitioned for further clinical development in sub-Saharan Africa. Since its inception the EVI organization has operated as a funding agency, but with a clear service-oriented strategy. The scientific successes and difficulties encountered during these years and how these efforts have led to standardization and harmonization in vaccine development through large-scale European consortia are discussed. In the future, the EVI will remain instrumental in the pharmaceutical and clinical development of vaccines against 'diseases of poverty' with a continued focus on malaria. EVI will continue to focus on funding and managing preclinical evaluation up to Phase I/II clinical trials and strengthening the vaccine-development infrastructure in Europe, albeit with a global orientation.

KEYwORDs: diseases of poverty $\bullet \mathrm{EMVI} \bullet \mathrm{EVI} \bullet$ malaria $\bullet \mathrm{PDP} \bullet$ research infrastructure $\bullet$ vaccine

Diseases of poverty (DoP) are a massive public health burden in many of the most resourceconstrained populations of the world [1]. In the context of the Millennium Development Goals, several governmental development agencies have committed themselves to reducing the burden of poverty related diseases, such as malaria. This commitment has been translated into support for academics and not-for-profit product development partnerships (PDPs), through technical and operational support and day-to-day project management.

The European Vaccine Initiative (EVI) and its predecessor the European Malaria Vaccine Initiative (EMVI) has acted as a partner to bring together public development organizations and institutions involved in the design, development and testing of novel vaccines. EVI not only directly funds the early stage development of vaccine candidates, but acts as a focal point for European and global vaccine research and development activities by coordinating numerous externally funded consortia that are working towards accelerating the development of safe, effective and affordable vaccines.

This review, by addressing the past and current portfolio, will illustrate how strategies of
EVI's vaccine development have evolved over the last decade through the lessons we learned from our successes and failures. Finally, we will cast an eye to the future.

\section{Past}

The genesis of EMVI dates back to February 1997 when the European Commission (EC)'s International Cooperation Research Programme with Developing Countries (INCO DC) submitted a proposal to the EU Member States. The proposal was fuelled by years of separated national and EC funding in the development of a malaria vaccine and a desire to improve communication and delivery between these separate programs. At the outset, EMVI's scope of operations addressed the translational gap between potential malaria vaccine candidates developed through basic science, and limited industrial production and early phase clinical trials (CTs). This niche, while being technically and legally complex, had traditionally been under financed.

Malaria was and is still among the most important parasitic cause of death and suffering today [101]. It is a major public health problem, particularly in sub-Saharan Africa, where the majority of the estimated annual cases of malaria 
occur. Each year worldwide, it is estimated that there are over 250 million cases of malaria with over one million deaths. The overwhelming proportion (85-90\%) of the malaria-attributable deaths occurs mainly in vulnerable populations of children under 5 years and pregnant women living in the endemic areas of subSaharan Africa [2-4]. Increased funding has supported the scale-up of malaria-control measures using the current control tools, such as insecticide-treated bed nets, procurement and distribution of anti-malarial drugs, early detection and treatment of malaria cases and vector control $[5,101]$. This has resulted in small but significant decrease in malaria incidence and prevalence in a limited number of countries where malaria is endemic.

There is no doubt that the key tool missing from the malaria control toolbox is an effective vaccine that would assist in the reduction or even elimination of the malaria disease [6]. The search for this tool has been ongoing for many decades and has included a myriad of vaccine concepts which until today have yielded only one partially efficacious ( $<50 \%$ efficacy) malaria vaccine, RTS,S, developed by a Malaria Vaccine Initiative (MVI) and GlaxoSmithKline Biologicals (GSK Bio; Rixensart, Belgium) collaborative effort [7-9]. This encouraging but less than satisfactory result suggests the need for continued efforts to develop the next generation of highly efficacious malaria vaccines $(>80 \%$ efficacy).

Three main scientific hurdles have impeded the successful development of an efficacious malaria vaccine:

- The enormous complexity of the parasite and its life cycle. We have limited knowledge of the interaction between the parasites and the human immune system. The parasite has been able to coevolve with its host and has thus developed strategies to evade the host's immune response;

- $\quad$ Extensive antigenic variation sensu stricto of the parasite;

- $\quad$ The different parasite lifecycle stages have unique antigen expression profiles.

Understanding the correlates of protection associated with the malaria infection and disease would support the development of an efficacious vaccine [10]. Although more knowledge has being gained from widespread and extensive studies and CTs in these areas in the last few years, there is still lack of full understanding of all these complex issues [6,11].

In comparison to other diseases where no correlates of protection are known (e.g., HIV) malaria infection can provide clinical immunity. Previously published reviews have extensively discussed this naturally acquired immunity to malaria [12], which is acquired slowly after a number of exposures but has a relatively short duration. A better understanding of the host's defense mechanisms and the Plasmodium targets that provide protection from malaria disease will go a long away in the advancement of the knowledge required to develop a vaccine that will reproduce this natural immunity.

\section{Vaccine portfolio}

EVI actively participated in the development of the Malaria Vaccine Technology Roadmap (MVTR) [102] and since then, all EVI's vaccine development efforts have followed this recommended strategy. The roadmap contains the views and vision of malaria
Figure 1. European Vaccine Initiative-funded projects and status at the end of 2010. EDCTP: European and Developing Countries Clinical Trials Partnership; EMVDA: European Malaria Vaccine Development Association; EVI: European Vaccine Initiative; GMP: Good manufacturing practice.

Reproduced with permission from [112]. 
experts toward the development and utilization of effective malaria vaccines. EVI supports malaria vaccine candidates that emerge from academic laboratories by sourcing and providing expertise and funding for production in line with Good Manufacturing Practice (GMP) and early clinical testing of the malaria vaccine candidates. EVI funding is targeted at diseases that may not be addressed by the pharmaceutical industry owing to likely poor economic returns. An overview of the EVI product portfolio and vaccine formulations is shown in Figure 1 and has been previously described [13].

\section{Subunit vaccines}

AMA1

In collaboration with the Biomedical Primate Research Centre (BPRC), two Phase Ia/Ib CTs of apical membrane antigen 1 (AMA1) adjuvanted in aluminum hydroxide, SEPPIC's Montanide ISA 720 and GSK's AS02 were completed in Nijmegen, The Netherlands and in Bandiagara, Mali. In both CTs, the blood-stage vaccine formulations showed acceptable safety and immunogenicity profiles. In Phase Ia, in vitro growth inhibition assay (GIA) showed levels of up to $80 \%$ antibody activity [14]. However, in the Phase Ib in Mali, although AMA1-specific IgG levels rose in vaccinees, the vaccine did not appreciably change GIA titers (Figure 2) [Thera M, Unpublished $D_{\text {DATA }}$. Thus, one of the conclusions of this clinical trial was that the polymorphisms in the AMA1 protein need to be addressed for the vaccine to show possible high efficacy in the field. This is discussed below in the section entitled AMA1-DiCo. These results were comparable to another AMA1 vaccine based on the 3D7 and FVO strains of Plasmodium falciparum, developed by National Institute of Allergy and Infectious Diseases, and the epidemiological data from Kenya [15,16]. The discontinuation of AMA1 is an example of project which did not fulfill the EVI go/no-go criteria.

\section{GMZ2}

GMZ2 is a recombinant fusion protein of two $P$. falciparum proteins, glutamate rich protein (GLURP) and merozoite surface protein 3 (MSP3), which EVI picked up in 2002 for development [17]. Following a successful Phase Ia CT at the University of Tubingen (Germany) in 2006/2007, two subsequent Phase Ib CTs in African adults and children were conducted at the Albert Schweitzer Hospital, Lambaréné, Gabon [18,19]. Results from these CTs showed that the GMZ2 blood-stage vaccine candidate, three doses of which were administered 4 weeks apart, was well tolerated and induced satisfactory immune responses. Subjects who received the GMZ2 vaccine candidate showed a 1.4-fold $(95 \%$ CI: 1.1-1.7) higher baseline-corrected anti-GMZ2 antibody levels and more GMZ2-specific memory B cells compared with the control group [19]. Unfortunately, the development of the GMZ2 vaccine concept was hampered because the mechanism of its function could not be sufficiently elucidated by the read-out of CT samples through the Antibody Dependent Cellular Inhibition (ADCI) assays. The necessity to have standardized and harmonized ADCI assays led to the development of the EC-funded OPTIMALVAC project (see below).

Currently, a Phase IIb multicenter efficacy CT in children aged $1-5$ years is currently ongoing at four CT sites in sub-Saharan

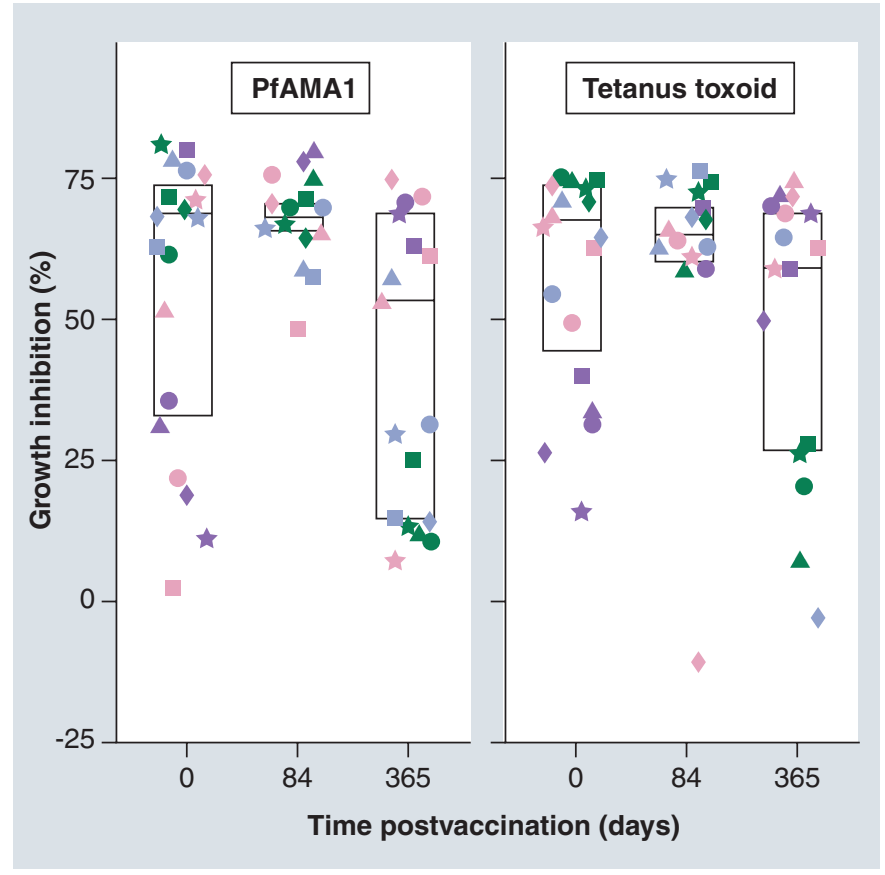

Figure 2. Growth inhibition assay titers in PfAMA1 vaccinated and control subjects (intention to treat) at $10 \mathbf{~ m g / m l}$ total IgG. Individual data points are plotted as well as a box indicating median and 25 and $75 \%$ quintiles.

Data taken from [Thera M, Unpublished Data].

Africa. EVI has managed to transfer this project to the European and Developing Countries Clinical Trials Partnership (EDCTP), contributing by providing the current GMP batch. This CT is being conducted under the auspices of the EDCTP-funded GMZ2 Consortium [103]. Results from this CT are awaited in 2011/2012.

\section{Polyprotein}

In 2002, EVI funded the assessment of the safety, immunogenicity and efficacy of two poxvirus malaria subunit vaccine candidates, FP9-PP and MVA-PP, which express the same polyprotein and consist of six pre-erythrocytic antigens from $P$. falciparum. Although vaccine candidates were safe, the immune responses induced by vaccination were low compared with vaccine candidates of a similar nature. No efficacy was observed on sporozoite challenge. Definite reasons for these unsatisfactory results are unknown but possible explanations for the lack of immunogenicity in CTs have been recently outlined [20].

While there is now increasing agreement that multiple antigens and multiple stages will be required in a highly efficacious vaccine, this polyprotein approach, which was designed to broaden the immune responses induced by these vaccines, appear to have been unsuccessful. There is a suggestion that there may be a limit to the size of the inserts (in this case polyprotein transgene) that will be immunogenic in humans.

Following the evaluation of these results, other vaccine-administration strategies, including the mixture of various single component vaccines are now being tried as alternatives in current preclinical development of these groups of vectored vaccines. 


\section{Table 1. Assessment criteria for development of malaria vaccines: rationale for choice of antigen.}

\section{Attribute}

Stage of parasite

Accessibility

Functionality

In vivo protection after immunization with the vaccine antigen in an animal model

In vitro inhibition

Sequence diversity

Parasite dependence on antigen

Epidemiological association of antigen with protection

Antigenicity

Pre-existing clinical data

IFA: Immunofluorescence assay.

Reproduced with permission from [104].

\section{MSP3}

In 1998, the safety and immunogenicity of the antigen MSP3 produced as a long synthetic peptide (LSP) was tested in a Phase Ia CT. MSP3-LSP was formulated in two adjuvants, aluminum hydroxyde and Montanide ISA 720. There were unacceptable local reactions with the MSP3-LSP plus Montanide ISA 720 after the second dose, which led to the withdrawal of some volunteers. MSP3-LSP was immunogenic with both adjuvants but the unacceptable reactogenicity with the Montanide adjuvant led to the decision not to take the MSP3-LSP plus Montanide ISA 720 formulation further in planned CTs in Africa. A recent review has shown that malaria antigens as LSP are immunogenic in human CTs [21].

The collapse of the small and medium-sized enterprise responsible for GMP production of MSP-LSP (Dictagene S.A., Lausanne, Switzerland) led to the discontinuation of EVI funding for this project. However, further development of this vaccine candidate was taken up by AMANET/Institut Pasteur (Paris, France) and recent publications from the Centre National pour la Recherche et la Formation sur le Paludisme (CNRFP, Burkina Faso) and the Institut Pasteur show that progress has been made with this vaccine formulated in aluminum hydroxide $[22,23]$. In a limitedsize CT in children at 1-2 years of age, the incidence of malaria was lower in the MSP3 vaccinated children than in the control group, at 60 days after the last immunization. Those preliminary results need to be confirmed in a larger size CT but support the development of a blood-stage vaccine for preventing malaria.

\section{EURHAVAC}

From the lessons which were learned during the initial malaria vaccine development efforts (1998-2004), the European Network for Harmonisation of Malaria Vaccine Development (EURHAVAC), an EC 6th Framework Programme (FP6), was initiated. In collaboration with the WHO and the malaria vaccine community, the consortium focused on the formulation of decision-making processes for supporting the development of more innovative and rationale-based vaccines that are carefully guided through design and production into CTs. In TABLE 1 an example is given of antigen assessment criteria for evaluation of potential malaria vaccine candidate antigen concepts prior to development. These go/no-go criteria are still used by the EVI. Four other categories of assessment criteria were formulated: vaccine rationale, vaccine delivery systems and formulation, antigen/vaccine production processes, and antigen and vaccine characterization. These criteria are available on the internet [104].

\section{Present}

During the last decade, EVI has demonstrated its ability to establish and maintain an enabling environment that allows malaria vaccine development: it has funded 29 different vaccine 
adjuvant/delivery formulations resulting in 13 vaccine candidates advanced into Phase I CTs.

The current EVI product portfolio currently comprises the following vaccines.

\section{Subunit vaccines}

AMA1-DiCo

Using knowledge generated from earlier CTs indicating allelic breakthrough, three artificial AMA1 sequences with a very high coverage of naturally occurring alleles were designed (on average $>97 \%)$. This diversity covering ( $\mathrm{DiCo}$ ) approach is currently under development by a continuing EVI-BPRC collaboration and is expected to overcome the polymorphisms found in nature. For the AMA1-DiCo vaccine candidate, process development and adjuvant selection have been completed and preparations for a Phase Ia and Ib CTs in European and African adults are ongoing.

During the development of the AMA1 concept with different adjuvants (aluminum hydroxyde, Montanide ISA 720 and ASO2), it became evident that divergence existed with respect to vaccine safety evaluation. These developments illustrated the necessity to further standardize vaccine safety evaluation. A second important lesson from the development of the AMA1 concepts was the difficulty in comparing the clinical settings in which the different AMA1 formulations were previously tested in the world by, for example, US groups and Australian groups [24]. The collaboration of the Malaria Vaccine Funders Group (MVFG) led to the selection of exactly the same CT site in Mali for the BPRC CT and further necessitated the need for harmonization of clinical development strategy for malaria vaccine candidates developed in Europe, including definition of the go/no go criteria for entering Phase Ia CTs, definition of safety evaluation criteria and definition of immunological evaluation criteria [25].

\section{P27A}

EVI supports one innovative peptide blood-stage vaccine candidates against malaria. The first approach comes from Corradin and collaborators who have demonstrated that a bioinformatics/ chemical synthesis approach can lead to the rapid identification of molecules that target biologically active antibodies. They selected several $\alpha$-helical coiled-coil domains of the proteins predicted to be present in the parasite erythrocytic stage [26]. A novel malaria blood-stage vaccine candidate, the $P$. falciparum 3D7 protein PFF0165c, also known as P27A, was identified by this process [27]. Since 2010, EVI has supported the preclinical assessment of this antigen. Process development and GMP production of this vaccine concept are currently ongoing.

\section{JAIVAC-1}

The malaria vaccine candidate JAIVAC-1 of the International Centre for Genetic Engineering and Biotechnology, (ICGEB [India, New Delhi]), is currently undergoing a proof-of-concept Phase I safety and immunogenicity CT. This vaccine is a combination of amino-terminal, conserved, cysteine-rich region of the $175-\mathrm{kDa} P$. falciparum erythrocyte-binding antigen EBA175 and 19-kDa MSP-1, adjuvanted in Montanide ISA 720 [28,29]. Enrolment and vaccinations have has been completed and the $\mathrm{CT}$ is expected to end in the fourth quarter of the calendar year (Q4) 2011.

In recent years, it has become widely accepted within the malaria vaccine community that deploying Montanide ISA 720 as an adjuvant in malaria vaccine formulations for children in Africa will be problematic. In parallel to the ongoing proof-of-concept Phase I CT, ICGEB has made considerable efforts to provide a suitable adjuvant for enhancement of the safety and immunogenicity of the JAIVAC-1 vaccine. Subsequently, collaboration is ongoing with the Infectious Disease Research Institute (IDRI) and the TRANSVAC consortium to meet this objective.

\section{Vectored vaccines}

The CSVAC project focuses on the development of an experimental circumsporozoite protein vectored vaccine against malaria, prepared using the chimpanzee adenovirus 63 vector (ChAd63) [30]. This pre-erythrocytic vaccine candidate is currently undergoing GMP production and a Phase I CT is planned to start in Q4 2011. The ChAd63 vector has several advantages:

- Lack of significant pre-existing immunity to ChAd63 in most human populations [Hill A, Unpublished Data];

- High yield of viral particles in a GMP manufacture construct;

- High immunogenicity of ChAd63 recombinants in animal models, matching or exceeding that of the potent Ad5 recombinant vector [31];

- Good safety and encouraging immunogenicity in an ongoing Phase I CT of this vector with another malaria insert (ME-TRAP).

The rationale to develop this vector in combination with CSP while the RTS,S concept is already in late-stage development is built upon extensive preclinical data in murine models that clearly show that very high levels of CD8 $\mathrm{T}$ cell are required to provide protective efficacy at the liver stage of infection with the CSP antigen. RTS, S induces minimal detectable CD8 T-cell response in humans [32] and first- and second-generation vectored vaccines, such as DNA-MVA produced only modest CD8 T-cell immunogenicity in humans [33]. However, new simian adenovirus vectors induce potent $\mathrm{CD} 8 \mathrm{~T}$ cells in humans against other malaria antigens in Phase I/II CTs [34,35] and so there is a strong prospect that this new prime-boost strategy will be more effective with CSP.

The clinical development of viral vectored malaria vaccine candidates is also funded by an EDCTP grant to the EVI-coordinated Malaria Vectored Vaccines Consortium (MVVC) [105]. This 4 -year project was set up with the aim of integrating capacitybuilding and networking in the design and conduct of Phase I and II CTs of viral-vectored malaria vaccine candidates in East and West African adults, children and infants. The research objective of the MVVC is to demonstrate safety and immunogenicity of an adenoviral vector modified Vaccinia Ankara (MVA) virus primeboost regime, encoding ME-TRAP in adults and young children in sub-Saharan Africa. The pre-erythrocytic vaccine candidates to 
be evaluated are ChAd63 ME-TRAP and MVA ME-TRAP. Age de-escalating assessment of the safety and immunogenicity of this new prime-boost regime in protection against clinical malaria in adults, 2-6 year-old and 5-17-month-old children has started in two African CT sites located in East and West Africa and evaluation of efficacy will be conducted in three other African CT sites.

As predicted from preclinical studies, the preliminary results from the ongoing CTs show that the heterologous prime-boost malaria vaccine regimens ChAd63 ME-TRAP and MVA ME-TRAP are safe and considerably more immunogenic than previous vectored vaccines in semi-immune Gambian adults and children aged 2-6 years. Results from efficacy CTs are expected in Q4 2013.

\section{European Malaria Vaccine Development Association}

In addition to directly supporting malaria vaccine activities, in 2006 EVI along with 19 other scientific institutions in Europe and Africa successfully applied to the EC FP6 for a grant for the development of malaria vaccine candidates. EVI plays a leading role as the coordinator of this integrated project, referred to as the European Malaria Vaccine Development Association (EMVDA) [106], run in partnership with all European institutions developing blood-stage malaria vaccines. A scientific and technological structure has been established to move malaria vaccine candidates from preclinical to early phase clinical development. EMVDA has provided a platform for scientists to compare the expression of the antigens in different vector-based systems, or the enhancement of the immunogenicity by different adjuvants. All the partners agreed to use the same stringent go/no-go criteria, to decide which formulation will be then tested in humans. These criteria include continuous evaluation as to whether scientific, evidence-based rationales (efficacy, technical and regulatory feasibility) are present that justify further development [36]. This strategy ensures that resources are allocated in the most efficient way to move the most promising vaccine candidates into GMP production and Phase I CT. Currently, four vaccine candidates have been selected: MSP1 prime-boost with ChAd63 + MVA and AMA1 prime-boost with ChAd63 + MVA (both University of Oxford, UK), full-length MSP1 (Heidelberg University, Germany), SR11.1 (Centre Hospitalier Universitaire Vaudois, Switzerland). To select and promote development of the most promising vaccine candidates, EMVDA has united the capabilities of small and medium-sized enterprises, major European malaria vaccine research centers, EVI, and African partners already linked through the AMANET as a consortium.

The EMVDA project is organized into six areas:

- Candidate antigens;

- Platforms and adjuvants;

- Vaccine development including assays for vaccine evaluation;

- Demonstration activities including GMP production and Phase I CTs;

- Training, integration and partnership activities;

- Management activities comprising both consortium management and product-development management.
The structure of EMVDA was based on lessons learned from the EURHAVAC consortium [25]. The EMVDA consortium actively cooperates with other global stakeholders in the field of malaria vaccine development, and in particular WHO. It participates in, and contributes to, agreed global coordination and harmonization efforts [102].

\section{Vaccine harmonization, coordination \& capacity strengthening}

Every vaccine being developed has its own technical and societal characteristics that renders the vaccine unique. However, to avoid loss of, and contribute to, harmonization of critical technological knowledge, the transfer of this knowledge needs to be secured, both within and outside the EU. To this end, EVI has coordinated the research networks EURHAVAC, PHARVAT, INYVAX and OPTIMALVAC, which are specific EC FP6-funded actions on harmonization of vaccine development in Europe and beyond.

PHARVAT aims to generate a harmonized procedure to permit preclinical selection of vaccine adjuvants throughout Europe and will finish by the end of 2011. The overall goal of OPTIMALVAC is to develop harmonized assays for parasite-antigen recognition, antibody and cell-dependent parasite inhibition and cell-mediated immune-response assays. The consortium currently focuses on the development, dissemination and implementation of Standard Operating Procedures for the conduct of key assays to Good Laboratory Practice level. To meet all of this, the consortium has set its goals on the development and sharing of reference preparations and common reagents for ADCI, ELISA, immunofluorescence assay (IFA) and GIA [37]. Furthermore, OPTIMALVAC is establishing a web-based tool for communication and tracking of activities. Consistent, reproducible and comparable intra- and interlaboratory performance and increased accuracy and precision of assay data will strengthen the quality of the vaccine development and CTs and will generate greater confidence in the evaluation of potential malaria-vaccine candidates.

The overall goal of INYVAX is to improve access to vaccine technology spanning across different diseases by developing knowledge-based platforms, that is, for antigen discovery optimization of delivery systems and formulations. In addition, INYVAX is developing safety standards in prelicensure CTs of vaccines against DoP, allowing meta-analysis of the safety of similar vaccine approaches [38]. Finally, it provides funding for advanced training in vaccinology for vaccine researchers on the ADVAC course.

When EVI funds the development of vaccine candidates, it ensures training and assurance in Good Clinical Practice (GCP), and in project management, including training of personnel from the endemic areas. In addition, over the 13 years of its existence, EVI has supported $12 \mathrm{PhD}$ students through its own grants or via EC-funded consortia.

\section{TRANSVAC}

Europe's position as the leader in different fields of vaccinology is now hampered by the decision of European governments, for economic reasons, to withdraw from funding state-owned 
vaccine production facilities [39]. This withdrawal of funding is likely to slow down innovation in vaccine research, having an immediate effect on the pool of knowledge and competencies, and increasing the degree of fragmentation of know-how and facilities. Infections do not stop at borders: the vaccine industry is global, but in Europe vaccine research institutes are national. In the current world economic and political situation there is an obvious absence of a European agenda on vaccine $\mathrm{R} \& \mathrm{D}$ both in terms of production and number of workers. In addition, there is a lack of synergy between the private and the public sector, and lack of commonly defined strategies by the EU Member States' public sectors, with a limited counterweight to the heavy policy influence of the private sector. The European vaccine development community needs to establish an efficient, sustainable collaborative infrastructure based on shared visions and goals in order to address these challenges. Through EVI, TRANSVAC, a collaborative infrastructure project funded under the EC FP7, has emerged as a joint effort of leading European groups in the field of vaccine development to enhance coordination between vaccine $\mathrm{R} \& \mathrm{D}$ groups, assay developers and vaccine producers in Europe, and to propose a European road map for vaccine R\&D.

TRANSVAC aims to accelerate the development of promising vaccine candidates by bridging the gap between bench research and CTs. To reach this goal, TRANSVAC carries out activities at various levels:

- Research: fostering the development of experimental vaccines through improving the use of (molecular) assays, standardized reagents, adjuvants, animal models, and antigen and cell bank production;

- Networking: the consortium provides training in vaccine development, harmonizing assays and microarrays, and optimal animal models. Activities here include a modular course on concepts in vaccine development as well as a series of workshops on animal models;

- Services: providing researchers with free access to adjuvant formulation, animal models, standards and global analyses (e.g., microarrays). These services are at the core of the consortium and are free of charge to European vaccine R\&D groups.

Through a peer-reviewed competitive process, European groups working in vaccine development may apply to benefit from the expertise, reagents and facilities of the TRANSVAC consortium. TRANSVAC's vaccine development services are not linked to any particular type of disease. Currently, 11 proposals have been funded through TRANSVAC services and they can be found on the TRANSVAC website [107].

\section{Future}

\section{Lessons learned from malaria vaccine development}

The EVI has evolved considerably over the last few years. In the past, EVI has focused on the translation of basic vaccine research, with a goal to bridge the gap between bench and bedside through the validation of vaccine concepts and supporting the transition of academic knowledge into products within a regulatory framework. However, when innovation in research is not stimulated enough, then very few new vaccine candidates are available for proof-of-concept, that is, the EVI pipeline is drying up. In Europe it has classically been the role of the national research councils and of the Directorate General (DG) research of the EC to fund innovation. The risk associated with this has become evident over the last few years and to mitigate this risk it has been decided by the EVI Scientific Advisory Committee to broaden the strategy and commence funding of vaccine concepts and technical platforms that are still in the discovery phase of development. The vaccines developed by EVI must be safe, efficacious and affordable.

\section{Safety}

EVI has been facing several safety challenges when developing new antigens with new adjuvants, during toxicological studies (GMZ2 with DDA-TDB) [108] or during Phase I CTs [14]. Recommendation is now made to the scientists to first enhance the antigenicity of their molecules and to preferably consider already validated adjuvants for CTs. Standardized assessment of safety during CTs is being implemented following the INYVAX guidelines, developed by the Brighton Collaboration.

\section{Efficacy}

An effective malaria vaccine is possible, as shown by the development of the partially efficacious RTS,S vaccine by GSK Bio [40]. In 2010, more than a dozen vaccines had reached the stage of Phase II CTs, nine were tested in human challenge CTs, three of them in partnership with EVI, five vaccines were in Phase IIb, two of them in partnership with EVI [108]. In a Phase I CT in children, the use of the MSP3 vaccine has demonstrated a reduction of the incidence of malaria episodes [22]. There are no correlates of protection available; therefore human challenge CTs should be an important gateway in the screening of new antigens. Through EMVDA, EVI is participating in the development of blood-stage human challenge model.

\section{Affordability}

The choice of producing the subunit antigens as long synthetic peptide vaccines and not as recombinant vaccines was decided because of the low price of GMP production. After several technical challenges, MSP3 is in Phase II CTs, and P27A will be also a synthetic vaccine. The high cost of production of the RTS,S vaccine is mainly due to the complex adjuvant that is needed to elicit immune response and protection. EVI will investigate new approaches to enhance the immunogenicity of the antigens, thus supporting new delivery and presentation systems.

\section{Accelerating the vaccine development}

EVI has proven through its development of the GMZ2 and MSP3 blood-stage vaccine candidates that it can facilitate vaccine development through to Phase II clinical testing. Both concepts were deemed safe and tested in dose-finding CTs within $3-6$ years. 
Together with its partners, EVI has witnessed the concerted evolution from the classical way of conducting Phase Ia CTs in Europe and subsequent Phase Ib CTs in Africa towards multicenter combined Phase I CTs. This trend has reduced both lead times and costs. Simultaneously, human challenge studies have matured and proved their value. One of the next key steps is the transfer and establishment of the human challenge model to clinical research centers in sub-Saharan Africa.

However, to date, the subunit malaria vaccine candidates have not yielded the aspired high vaccine efficacy. This has fuelled the development of testing of vaccination with whole-cell preparations, either as irradiated, live-attenuated cells or as a low dosage of blood-stage parasites [41-43]. Once injected, the sporozoite stage of the malaria parasite confer long-lasting, sterile protection against infection, providing a benchmark for vaccine development [44]. Furthermore, the discovery of genetically engineered parasite strains that are fully attenuated during the liver-stage infection and confer sterile protection in animal malaria models [42] support the development of a live-attenuated sporozoite vaccine for $P$. falciparum and its accelerated safety and efficacy testing in malaria challenge models and in malaria endemic areas represents a significant step forward in malaria vaccine development.

\section{Neglected tropical diseases}

The WHO estimates that more than 1 billion people living on $<$ US $\$ 2 /$ day are affected by at least one 'neglected tropical disease' (NTD) [109]. NTDs are flourishing in low-income populations and are an obstacle for the achievement of health in the Millennium Development Goals. Their significance to public health and economies is obvious and grouping them under one term can ensure concerted and focused actions for governments, non-governmental organizations, donors, industry and international agencies.

For vaccine development against the three major DoPs: HIV, TB and malaria, highly focused product development initiatives already exist. Relative to other NTDs, which are not yet fully on the agenda of international or governmental development agencies despite their impact on low-income populations, these three diseases have attracted more funds than ever before. This has indeed created unprecedented pipelines with promising vaccine candidates for HIV/AIDS, TB and malaria. The pitfall would be to remove these three diseases, together still causing a lot of disease and death, from the list of neglected infectious diseases. Keeping the pipeline filled and bringing these vaccines successfully to the market at affordable prices will still demand huge amounts of public and philanthropic funding while industry is focusing on more profitable markets for survival. At the same time it would be tragic if this funding would come at the cost of sufficient financing for R\&D of the other NTDs, which some therefore term the 'really neglected' tropical diseases. It is in this niche that EVI will be supporting the development of vaccines. Vaccines are key assets in the fight against DoP, and therefore, accelerated development of safe and efficacious vaccines represents a top priority and should count on sufficient, predictable and sustainable funding at national and international level.
According to WHO, NTDs are mainly parasitic and viral diseases, that is, buruli ulcer, Chagas disease (American trypanosomiasis), cysticercosis, dracunculiasis (guinea-worm disease), echinococcosis, leishmaniasis, leprosy, lymphatic filariasis and schistosomiasis. For treatment of these diseases, a very limited array of drugs exists, most often to be taken over a long period of time and with serious side-effects. An effective vaccine does not yet exist for any of the NTDs.

EVI intends to support the development of vaccines against NTDs in the same way as it has done within the malaria field. First, a shared vision and strategy among the most relevant international experts and stakeholders from disease endemic countries will be developed. Once the goals are set, EVI will focus on antigen selection, assay standardization and validation, sustainable process development and manufacturing in accordance with international quality requirements. Clinical and regulatory strategies will be developed to ensure coherent and consistent development based on strong rationale and harmonization of the evaluation criteria used for safety, immunogenicity and protection.

\section{Trends in funding}

Funding for malaria and NTDs vaccine research comes from a variety of sources: governmental donors, multilateral organizations (WHO, World Bank and EC), philanthropic foundations and industry.

It is difficult to establish a clear picture of the total amount of resources invested in malaria vaccine research. First, one cannot get a good insight into the total investments made by the pharmaceutical industry in malaria vaccine R\&D. Second, because malaria vaccine development builds on fundamental research findings from various disciplines. Fundamental research is coordinated through national research councils and financed with national public funds, resulting in a variety of national funding streams and incentives, thus fragmenting the research landscape.

Funding for malaria vaccine research has been on the rise since the mid-1990s. From an unknown proportion of a total of US\$84 million for malaria R\&D in 1993 (probably US\$20 million extrapolated from later figures for vaccine development), going up to US\$79 million for malaria vaccine R\&D in 2004 [110] and, despite the global financial crisis, rising further to US\$195.8 million in 2009 [111]. However, recent global economic and financial developments indicate that the impact of the financial crisis is leading to serious fiscal constraints. As a result many donors are drastically cutting budgets for international development cooperation and requiring that funding should serve national interests first [39].

It is important to see the current malaria vaccine funding in light of current progress in malaria vaccine development. The results of CTs with the RTS,S pre-erythrocytic malaria vaccine, developed by GSK Bio and funded by the Bill \& Melinda Gates Foundation, via the MVI, have generated renewed attention for malaria vaccines and have reinvigorated the conviction of scientists that a protective malaria vaccine is feasible.

In a recent article it was concluded that the RTS,S vaccine will only be partially efficacious and the real health impact of 
this first-generation malaria vaccine still needs to be assessed [40]. Although RTS,S represents an important milestone in malaria vaccinology, the funding needed for Phase IV of RTS,S also generates a difficult conundrum for the allocation of the existing scarce resources for malaria vaccine $\mathrm{R} \& \mathrm{D}$ : continuation and even increase of investments to develop a partially efficacious vaccine or investment in $R \& D$ of second-generation and other types of malaria vaccines that can be brought to the market by (other) industry players. Putting 'all eggs in one basket' might be an unwise choice for the future of malaria vaccine development. Funding of a diversity of malaria vaccines might be the soundest strategy to defeat such a 'shrewd' organism as the malaria parasite, but this comes at a cost.

Learning from experience and analyzing the latest global developments, four different perspectives are feeding into the EVI funding strategy which will focus on malaria and DoP:

- Combining public and private efforts;

- Convergence of national and international research objectives;

- Assembling a research infrastructure for long-term objectives;

- Enabling serendipity by multidisciplinary and cross-disease fertilization.

\section{International collaboration}

EVI has signed separate memorandums of understanding with the US Agency for International Development Malaria Vaccine Development Program, PATH Malaria Vaccine Initiative, Tuberculosis Vaccine Initiative, Brighton Collaboration Foundation and Malaria Vaccine Development Program and a memorandum of intent WHO. EVI is an active member of the MVFG, which was created in 2001, and has developed a global portfolio review and the MVTR [102]. Finally, the collaboration with the private sector must be strengthened, and the TRANSVAC project will play a major role in identifying and prioritising potential areas of collaboration with pharmaceutical industry, mainly the European Vaccine Manufacturers. Special attention will be paid to collaboration with the Developing Countries Vaccine Manufacturers Network (DCVMN). International cooperation is the only way the leverage of knowledge and resources can be achieved to combat DoP.

\section{Three scientific priorities of a sustainable vaccine development platform}

To be successful in the future in the development of new vaccines for malaria and DoPs, the focus of the EVI will be on what it calls the three scientific priorities of sustainable vaccine development platform. These three pillars are:

- Translational vaccine research;

- Knowledge sharing;

- Harmonization of vaccine research efforts.

\section{Translational research}

For the vaccine pipeline to be continuously filled it is imperative that creativity and innovation is fostered while remaining focused on the proof of concept, that is. early stages of clinical development, to ensure the realization of promising vaccine candidates that have the best possible chance of success. Although the basic science part of a vaccine is at the core of the concept, minimal levels of quality assurance, process development (analytical and process qualification), regulatory aspects and market entry should be taken into account in order to mitigate costly, late-stage changes during development. To this end, EVI facilitates technology transfer of production of new vaccine candidates to qualified producers. EVI works toward sustainability of its adjuvant platform, including access to new adjuvants and vaccine formulation, and encourages studies for better understanding of the mechanisms of adjuvant action. Likewise, it is necessary to keep on supporting innovative technology to improve the delivery of antigens (e.g., alternative routes of administration, improvement of heat stability and increase in yield). Finally, it is important to maintain a dialogue with policy makers through consultative working groups on specific scientific, regulatory or technical questions and sustained mobilization of funds for translational vaccine research.

\section{Knowledge sharing}

Next to ensuring that vaccine innovation can thrive, it is absolutely essential that knowledge created within vaccine development is disseminated appropriately. EVI aims to secure sharing of vaccine knowledge by the training of vaccine developers. In this context, EVI is expecting to set up a fellowship program for scientists interested in management of vaccine development. Additionally, EVI will continue to participate in training programs for project/program management and to conduct international workshops on scientific topics of relevance to vaccine development for DoP.

\section{Harmonization}

The harmonization of immuno-assays and clinical protocol development are key elements in generating comparative and reproducible results. The key focus should be that assay development is in line with new vaccine targets and provides real improvements in evaluating immune responses in target populations. Finally, and probably the most difficult, is that through harmonization we can encourage the development of biomarkers and other surrogates of protection.

A key development is demonstrating through TRANSVAC the importance of the integration of vaccine development activities in Europe, and the optimization of resources allocated by the public sector to the R\&D of vaccines. TRANSVAC will explore the genesis of a European Vaccine Development Infrastructure. TRANSVAC will launch a special call in 2012 inviting applications for external institutions to join and further complement and strengthen the consortium, if they are able to bring relevant experience/expertise for mutual benefit into TRANSVAC. Through TRANSVAC, a working group with pharmaceutical industry will be put in place in order to identify the synergies between private and public sector. This assessment will drive the process for designing the strategy of the European Vaccine Development 
Infrastructure, which should be created in continuation of the EVI, along with other stakeholders.

\section{Expert commentary \& five-year view}

EVI's scope of operation in the vaccine development pipeline is in the gap between vaccine candidates for DoP developed via basic science and early phase clinical development. EVI has supported both financially and technically, all vaccine development activities through:

- Antigen selection, assay standardization and validation, development of challenge models and development of CT protocols and finally the manufacture of promising malaria vaccine candidates;

- Clinical and regulatory development based on strong rationale and harmonization of the evaluation criteria used for safety, immunogenicity and protection;

- The performance of early phase clinical development by funding Phase I and when relevant, validated Phase II CTs of these vaccines in a limited number of CT centers in Europe.

In the next 5-10 years, EVI will address the current major gaps in vaccine development by;
- Broadening the funding to vaccines targeted at all DoP to ensure a pipeline as diverse as possible;

- Extending the initiative focus on P. falciparum to other Plasmodium species;

- Strengthening the vaccine R\&D infrastructure in Europe through infrastructure projects funded by EC.

Further strategic focus will give to knowledge sharing, harmonization of research and techniques, and fostering translational vaccine research. EVI will continue to partner with industry and/or public institutions to support the pharmaceutical scaleup and production of clinical batches for full clinical development, through its main activities including communication with stakeholders, advocacy and raising funds.

\section{Financial \& competing interests disclosure}

The authors have no relevant affiliations or financial involvement with any organization or entity with a financial interest in or financial conflict with the subject matter or materials discussed in the manuscript. This includes employment, consultancies, honoraria, stock ownership or options, expert testimony, grants or patents received or pending, or royalties.

No writing assistance was utilized in the production of this manuscript.

\section{Key issues}

- The European Vaccine Initiative (EVI) was initiated to address the translational gap between promising diseases of poverty vaccine candidates and early-phase clinical trials.

- The EVI supports all diseases of poverty vaccine development activities, both financially and technically.

- In the last decade, EVI has contributed to the development of 24 malaria antigens and performed ten clinical trials in Europe and Africa.

- The EVI fosters standardization and harmonization within European vaccine development efforts through several European Commission consortia.

- The TRANSVAC consortium stakeholder group, which contains European vaccine R\&D groups, funders, regulators and vaccine manufacturers, will develop a European road map for sustained vaccine R\&D.

\section{References}

Papers of special note have been highlighted as:

- of interest

•• of considerable interest

1 Hotez PJ, Fenwick A, Savioli L, Molyneux $\mathrm{DH}$. Rescuing the bottom billion through control of neglected tropical diseases. Lancet 373(9674), 1570-1575 (2009).

2 Breman JG, Egan A, Keusch GT. The intolerable burden of malaria: a new look at the numbers. Am. J. Trop. Med. Hyg. 64(Suppl. 1-2), iv-vii (2001).

3 Roca-Feltrer A, Carneiro I, Armstrong Schellenberg JR. Estimates of the burden of malaria morbidity in Africa in children under the age of 5 years. Trop. Med. Int. Health 13(6), 771-783 (2008).

4 Thwing J, Eisele TP, Steketee RW. Protective efficacy of malaria case management and intermittent preventive treatment for preventing malaria mortality in children: a systematic review for the lives saved tool. BMC Pub. Health 11(Suppl. 3), S14 (2011)

5 O'Meara WP, Bejon P, Mwangi TW et al. Effect of a fall in malaria transmission on morbidity and mortality in Kilifi, Kenya. Lancet 372(9649), 1555-1562 (2008).

6 A research agenda for malaria eradication: vaccines. PLoS Med. 8(1), e1000398 (2011).

- $\quad$ Describes the strategy of the Bill \& Melinda Gates Foundation for the eradication of malaria and how transmission-blocking vaccines will support the eradication. This raises the need for an alternative strategy to address the prevention of the morbidity and mortality.

7 Alonso PL, Sacarlal J, Aponte JJ et al. Duration of protection with RTS,S/AS02A malaria vaccine in prevention of
Plasmodium falciparum disease in Mozambican children: single-blind extended follow-up of a randomised controlled trial. Lancet 366(9502), 2012-2018 (2005).

8 Cohen J, Nussenzweig V, Nussenzweig R, Vekemans J, Leach A. From the circumsporozoite protein to the RTS,S/AS candidate vaccine. Hum. Vaccines 6(1), 90-96 (2010).

9 Regules JA, Cummings JF, Ockenhouse CF. The RTS,S vaccine candidate for malaria. Expert Rev. Vaccines 10(5), 589-599 (2011).

10 Greenwood B, Targett G. The mysteries of immunity to malaria. Lancet 377(9779), 1729-1730 (2011).

11 Crompton PD, Pierce SK, Miller LH. Advances and challenges in malaria vaccine development. J. Clin. Invest. 120(12), 4168-4178 (2010). 
12 Doolan DL, Dobano C, Baird JK. Acquired immunity to malaria. Clin. Microbiol. Rev. 22(1), 13-36 (2009).

13 Imoukhuede EB, Ventura R, Imbault N, Van Schooten H, Leroy O. European Malaria vaccine initiative: portfolio and perspectives for the future. Hum. Vaccin. 6(1), 146-150 (2009).

14 Roestenberg M, Remarque E, De Jonge E et al. Safety and immunogenicity of a recombinant Plasmodium falciparum AMA1 malaria vaccine adjuvanted with Alhydrogel, Montanide ISA 720 or AS02. PLoS One 3(12), e3960 (2008).

15 Osier FH, Weedall GD, Verra F et al. Allelic diversity and naturally acquired allelespecific antibody responses to Plasmodium falciparum apical membrane antigen 1 in Kenya. Infect. Immun. 78(11), 4625-4633 (2010).

16 Ouattara A, Mu J, Takala-Harrison S et al. Lack of allele-specific efficacy of a bivalent AMA1 malaria vaccine. Malar. J. 9, 175 (2010).

17 Theisen M, Soe S, Brunstedt K et al. A Plasmodium falciparum GLURP-MSP3 chimeric protein; expression in Lactococcus lactis, immunogenicity and induction of biologically active antibodies. Vaccine 22(9-10), 1188-1198 (2004).

18 Esen M, Kremsner PG, Schleucher R et al. Safety and immunogenicity of GMZ2 - a MSP3-GLURP fusion protein malaria vaccine candidate. Vaccine 27(49), 6862-6868 (2009).

19 Mordmuller B, Szywon K, Greutelaers B et al. Safety and immunogenicity of the malaria vaccine candidate GMZ2 in malaria-exposed, adult individuals from Lambarene, Gabon. Vaccine 28(41), 6698-6703 (2010).

20 Porter DW TF, Berthoud TK, Hutchings CL et al. A human Phase 1/2a malaria challenge trial of a polyprotein malaria vaccine. Vaccine 29(43), 7514-7522 (2011).

21 Olugbile S, Habel C, Servis C, Spertini F, Verdini A, Corradin G. Malaria vaccines the long synthetic peptide approach: technical and conceptual advancements. Curr. Opin. Mol. Ther. 12(1), 64-76 (2010).

22 Sirima SB, Cousens S, Druilhe P. Protection against malaria by MSP3 candidate vaccine. N. Engl. J. Med. 365(11), 1062-1064 (2011).

23 Sirima SB, Tiono AB, Ouedraogo A et al. Safety and immunogenicity of the malaria vaccine candidate MSP3 long synthetic peptide in 12-24 months-old Burkinabe children. PLoS One 4(10), e7549 (2009).
24 Remarque EJ, Faber BW, Kocken CH, Thomas AW. Apical membrane antigen 1: a malaria vaccine candidate in review. Trends Parasitol. 24(2), 74-84 (2008).

25 Craig AG, Holder AA, Leroy OY, Ventura RA. Malaria vaccines - how and when to proceed? Trends Parasitol. 25(12), 535-537 (2009).

26 Villard V, Agak GW, Frank G et al. Rapid identification of malaria vaccine candidates based on $\alpha$-helical coiled coil protein motif. PLoS One 2(7), e645 (2007).

27 Olugbile S, Villard V, Bertholet S et al. Malaria vaccine candidate: design of a multivalent subunit $\alpha$-helical coiled coil poly-epitope. Vaccine 29(40), 7090-7099 (2011).

28 Pattnaik P, Shakri AR, Singh S, Goel S, Mukherjee P, Chitnis CE. Immunogenicity of a recombinant malaria vaccine based on receptor binding domain of Plasmodium falciparum EBA-175. Vaccine 25(5), 806-813 (2007).

29 Richards JS, Stanisic DI, Fowkes FJ et al. Association between naturally acquired antibodies to erythrocyte-binding antigens of Plasmodium falciparum and protection from malaria and high-density parasitemia. Clin. Infect. Dis. 51(8), e50-e60 (2010).

30 Hill AV, Reyes-Sandoval A, O'Hara G et al. Prime-boost vectored malaria vaccines: progress and prospects. Hum. Vaccin. 6(1), 78-83 (2010).

31 Reyes-Sandoval A, Sridhar S, Berthoud T et al. Single-dose immunogenicity and protective efficacy of simian adenoviral vectors against Plasmodium berghei. Eur. J. Immunol. 38(3), 732-741 (2008).

32 Moorthy VS, Ballou WR. Immunological mechanisms underlying protection mediated by RTS,S: a review of the available data. Malar. J. 8, 312 (2009).

33 Dunachie SJ, Walther M, Epstein JE et al. A DNA prime-modified vaccinia virus ankara boost vaccine encoding thrombospondinrelated adhesion protein but not circumsporozoite protein partially protects healthy malaria-naive adults against Plasmodium falciparum sporozoite challenge. Infect. Immun. 74(10), 5933-5942 (2006).

34 Sheehy SH, Duncan CJ, Elias SC et al. Phase 1a clinical evaluation of the Plasmodium falciparum blood-stage antigen MSP1 in ChAd63 and MVA vaccine vectors. Mol. Ther. doi:10.1038/mt.2011.176 (2011) (Epub ahead of print).

35 O'Hara GA, Duncan CJA, Ewer KJ et al. Clinical assessment of a recombinant simian adenovirus chad63: a potent new vaccine vector. J. Infect. Dis. (2011) (In Press).

36 Moorthy VS, Kieny MP. Reducing empiricism in malaria vaccine design. Lancet Infect. Dis. 10(3), 204-211 (2010).

-. Analyzes the limited performance of the current holistic approaches for discovery of malaria vaccines and advocates for a more scientific rationale design of malaria vaccines.

37 Cavanagh DR, Dubois PM, Holtel A et al. Towards validated assays for key immunological outcomes in malaria vaccine development. Vaccine 29(17), 3093-3095 (2011).

38 Kisser A, Heininger U, Moorthy VS, Akanmori BD, Leroy O. Addressing the needs and gaps in safety assessment of vaccines during clinical trials in resource limited countries. Vaccine 29(24), 41734174 (2011).

39 Thogersen RL, Holder AA, Hill AV, Arnot DE, Imoukhuede EB, Leroy O. Comparative decline in funding of European Commission malaria vaccine projects: what next for the European scientists working in this field? Malar. J. 10(1), 255 (2011).

40 Olotu A, Lusingu J, Leach A et al. Efficacy of RTS,S/AS01E malaria vaccine and exploratory analysis on anti-circumsporozoite antibody titres and protection in children aged 5-17 months in Kenya and Tanzania: a randomised controlled trial. Lancet Infect. Dis. 11(2), 102-109 (2011).

41 Roestenberg M, Mccall M, Hopman J et al. Protection against a malaria challenge by sporozoite inoculation. N. Engl. J. Med. 361(5), 468-477 (2009).

42 Mueller AK, Labaied M, Kappe SH, Matuschewski K. Genetically modified Plasmodium parasites as a protective experimental malaria vaccine. Nature 433(7022), 164-167 (2005).

43 Epstein JE, Tewari K, Lyke KE et al. Live attenuated malaria vaccine designed to protect through hepatic CD8 ${ }^{+} \mathrm{T}$ cell immunity. Science 334(6055), 475-480 (2011).

44 Vaughan AM, Wang R, Kappe SH. Genetically engineered, attenuated whole-cell vaccine approaches for malaria. Hum. Vaccin. 6(1), 107-113 (2010).

\section{Websites}

101 WHO. World Malaria Report 2010. www.who.int/entity/malaria/world_ malaria_report_2010/ worldmalariareport2010.pdf 
$102 \mathrm{WHO} /$ Initiative for Vaccine Research (IVR). Malaria vaccine technology roadmap. 2006.

www.malariavaccine.org/files/Malaria Vaccine_TRM_Final_000.pdf

103 GMZ2 Consortium Official Website. www.amanet-trust.org/gmz2/

104 Assessment criteria for evaluation of malaria vaccine concepts prior to clinical lot production: rationale for choice of antigen. 2008.

www.euvaccine.eu/sites/default/files/ uploads/docs/Projects/2008_ EURHAVAC_criteria.pdf

105 Malaria Vectored Vaccine Consortium. www.malariavvconsortium.eu
106 EMVDA. www.emvda.org

107 TRANSVAC. www.transvac.org

108 Malaria Vaccine Rainbow Tables. www.who.int/vaccine_research/links/ Rainbow/en/index.html (Accessed 1 October 2011)

-. Currently these spreadsheets represent the most complete publicly available summary of malaria vaccine projects at advanced preclinical and clinical stages globally.

109 WHO. First WHO report on neglected tropical diseases. 2010. http://whqlibdoc.who.int/ publications/2010/9789241564090_eng. pdf
110 Malaria R\&D Alliance 2005 Global Malaria Funding Survey.

www.malariavaccine.org/files/MalariaRD_ Report_complete.pdf

111 "Staying the course? Malaria research and development in a time of economic uncertainty".

www.policycures.org/downloads/

Malaria\%20Research\%20and\%20 Development.pdf

112 European Vaccine Initiative: vaccine Development.

www.euvaccine.eu/portfolio/vaccinedevelopment 